\title{
Re: Intraoperative Optical Imaging and Tissue Interrogation During Urologic Surgery
}

\author{
Hsu M', Gupta M², Su LM², Liao JC'1,3 \\ 'Stanford University School of Medicine, Department of Urology, Stanford, California, USA \\ 2University of Florida College of Medicine, Department of Urology, Gainsville, Florida, USA \\ ${ }^{3}$ Veterans Affairs Palo Alto Healthcare System, Palo Alto, California, USA
}

Curr Opin Urol 2014;24:66-74. doi: 10.1097/MOU.0000000000000010.

\section{EDITORIAL COMMENT}

The era of minimally invasive surgery includes development of endoscopic, laparoscopic, and robotic-assisted surgery techniques. In contrast of these developments, there are still some problems such as lack of tactile sensation, small operative fields and disadvantage of white-light sight. Depending on the advances in modern imaging technologies, additional improvements could be obtained in diagnosis and surgical procedures. Photodynamic diagnostic technique (PDD) is a fluorescence-based optical imaging technology that uses photosensitive protoporphyrin analogues as contrast agents. PDD has been applied principally in bladder cancer through intravesical contrast administration. Other new technique is near-infrared fluorescence image-guided surgery (NIRF). Indocyanine green is a contrast agent for intraoperative imaging that absorbs and emits light in the near infrared portion of the electromagnetic spectrum. Actual studies have investigated the use of intraoperative NIRF imaging to enhance intraoperative decision-making in prostate cancer or renal cell cancer surgery. Optical coherence tomography is an optical biopsy technique that provides highresolution, real-time, cross-sectional imaging of tissues for cystoscopy in bladder cancer, evaluation of kidney morphology and renal masses or detection of extraprostatic invasion in prostate cancer and identification of the neurovascular bundle during radical prostatectomy. Nowadays, confocal laser endomicroscopy (CLE) with fluorescein as the contrast agent enables real-time in vivo microscopy with the highest spatial resolutions among the optical imaging technologies. In vivo CLE is valuable especially for the evaluation of bladder cancer patients. Moreover, cancer-specific contrast agents can be hopeful as a molecular imaging technique for diagnosis of cancer. In the future, intraoperative optical imaging techniques will be more usable during urologic surgery.

Fehmi Narter, MD, PhD

๑Copyright 2018 by the Association of Urological Surgery / Journal of Urological Surgery published by Galenos Publishing House. 